\title{
Switchable Multiwavelength Erbium-Doped Fiber Laser using a Multimode Fiber Bragg Grating and Photonic Crystal Fiber
}

\author{
Xinhuan Feng, ${ }^{1}$ Hwa-yaw Tam, ${ }^{1}$ and P. K. A. Wai ${ }^{2}$ \\ ${ }^{1}$ Photonics Research Center and Department of Electrical Engineering, The Hong Kong Polytechnic University Hung Hom, Kowloon, \\ Hong Kong SAR, China \\ ${ }^{2}$ Photonics Research Center and Department of Electrical Information Engineering, The Hong Kong Polytechnic University Hung Hom, \\ Kowloon, Hong Kong SAR, China \\ eexhfeng@polyu.edu.hk
}

\begin{abstract}
A switchable multiwavelength erbium-doped fiber laser with a multimode FBG and highly nonlinear PCF is demonstrated. Stable five-wavelength operation with $0.8 \mathrm{~nm}$ spacing was achieved. Switching between stable one- to four-wavelength operations is also demonstrated.

(C) 2006 Optical Society of America

OCIS codes: (190.4380) Nonlinear optics, four-wave mixing; (140.3510) Lasers, fiber
\end{abstract}

\section{Introduction}

Multiwavelength fiber lasers are useful light sources for wavelength-division-multiplexed (WDM) fiber communication systems, fiber sensors, and testing of optical instrument. A number of techniques have been proposed to realize switchable multiwavelength oscillations in erbium-doped fiber lasers (EDFLs) [1-3]. In this paper, we report a stable multiwavelength EDFL which employs a multimode fiber Bragg grating (MM-FBG) for multiwavelength operations. We achieved simultaneous lasing of five wavelengths with 0.8-nm spacing using the four-wave mixing (FWM) effects induced by the highly nonlinear photonic crystal fiber (HNL-PCF) placed in the laser cavity. The proposed laser can either operate in five-wavelength mode or wavelength switching modes by adjusting a polarization controller (PC). In wavelength switching mode, single, dual, triple, and quadruple-wavelength operations have been obtained.

\section{Configuration and Principle}

Figure 1 depicts the schematic of the configuration of the proposed laser. A commercial EDFA with an output saturation power of $500 \mathrm{~mW}$ provides the gain. Twenty meters of HNL-PCF is used to generate FWM effect to obtain stable multiwavelength oscillations. The HNL-PCF has a zero-dispersion wavelength around $1550 \mathrm{~nm}$ and a nonlinearity coefficient of about $30.6 \mathrm{~W} / \mathrm{km}$. An MM-FBG is introduced into the cavity by an optical circulator (OC) for multiwavelength selection. The PC is used to adjust the states of polarizations (SOPs) of the lights incident on the HNL-PCF. The fibers in the cavity are all singlemode fibers (SMFs) except for a 1-m section of multimode fiber (MMF) which contains the MM-FBG. The laser output was taken via the 10\% port of a 90:10 fused fiber coupler and was measured using an optical spectrum analyzer with $0.1 \mathrm{~nm}$ resolution.

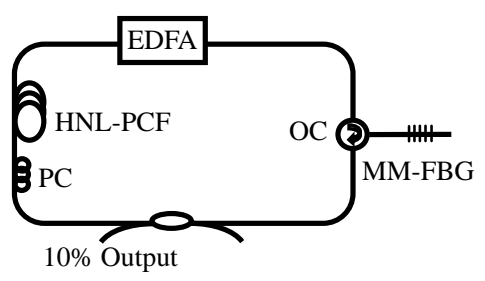

Fig.1. Schematic diagram of the proposed laser.

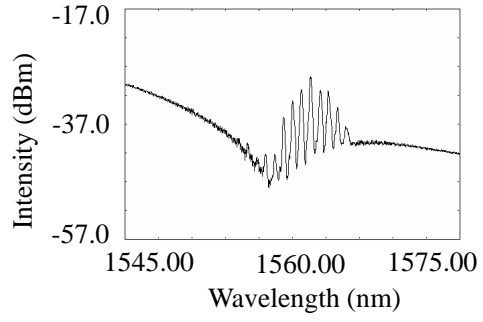

Fig.2. Reflection spectra of the MM-FBG.

In the experiment, the lateral displacement between the SMF and the MMF was varied so that several of the wavelengths reflected back by the MM-FBG can have approximately equal reflectivities (Fig.2). To achieve multiwavelength output, these wavelengths $\left(\lambda_{\mathrm{i}}\right)$ must simultaneously satisfy the oscillation conditions [4]:

$$
R_{i} r_{i} \cdot V_{i} \cdot G_{i E D F A} G_{i F W M}=1,
$$

Where $R_{i}$ and $r_{i}$ are the output coupler reflectivity and the FBG reflectivity for $\lambda_{\mathrm{i}}$, respectively, $V_{i}$ is the roundtrip losses in the fiber cavity, and $G_{i E D F A}$ and $G_{i F W M}$ are the gains obtained from the EDFA and FWM effect, 


\section{JThC47.pdf}

respectively. From Eq. (1), multiwavelength oscillation is possible if the FWM gain and the reflectivity of each wavelength are optimized such that the oscillation conditions are satisfied simultaneously. On the other hand, wavelength switching can be achieved if the oscillation conditions for different $\lambda_{\mathrm{i}}$ are satisfied at different FWM efficiencies. Since FWM efficiency depends on the SOPs of the input lights [5], we can vary the FWM efficiencies by adjusting the PC. Thus the FWM gain $G_{i F W M}$ at different wavelengths can be adjusted to satisfy the oscillation conditions for different $\lambda_{\mathrm{i}}$, and consequently both multiwavelength oscillation and wavelength switching operation can be obtained. From Eq. (1), it is possible to realize multiwavelength oscillation and wavelength switching by adjusting the reflectivities of the MM-FBG at different wavelengths. That is, adjusting the offset between the SMF and the MMF can also lead to wavelength switching, but this switching method is not as easy and convenient as using the PC.

\section{Results and discussions}

In the experiment, by adjusting the offset between the SMF and the MMF, and the states of the PC, simultaneous five-wavelength oscillations were obtained. Figure 3(a) shows the output spectrum. The five wavelengths are at 1563.21, 1562.42, 1561.61, 1560.80, and $1560.01 \mathrm{~nm}$ respectively. They have a wavelength spacing of $0.8 \mathrm{~nm}$. The inset in Fig.3(a) shows the power fluctuation of the filtered output at $1560.80 \mathrm{~nm}$ versus time. The maximum power fluctuation is $0.75 \mathrm{~dB}$. Then, using the same offset, by carefully adjusting the states of the PC, single-wavelength, dual-wavelength, triple-wavelength, and quadruple-wavelength operations have been obtained. Figure 3(b) shows one typical four-wavelength output spectrum. The outputs exhibit good performances having OSNR over $55 \mathrm{~dB}$ and the output power larger than $60 \mathrm{~mW}$ in all the operation conditions we have tested.
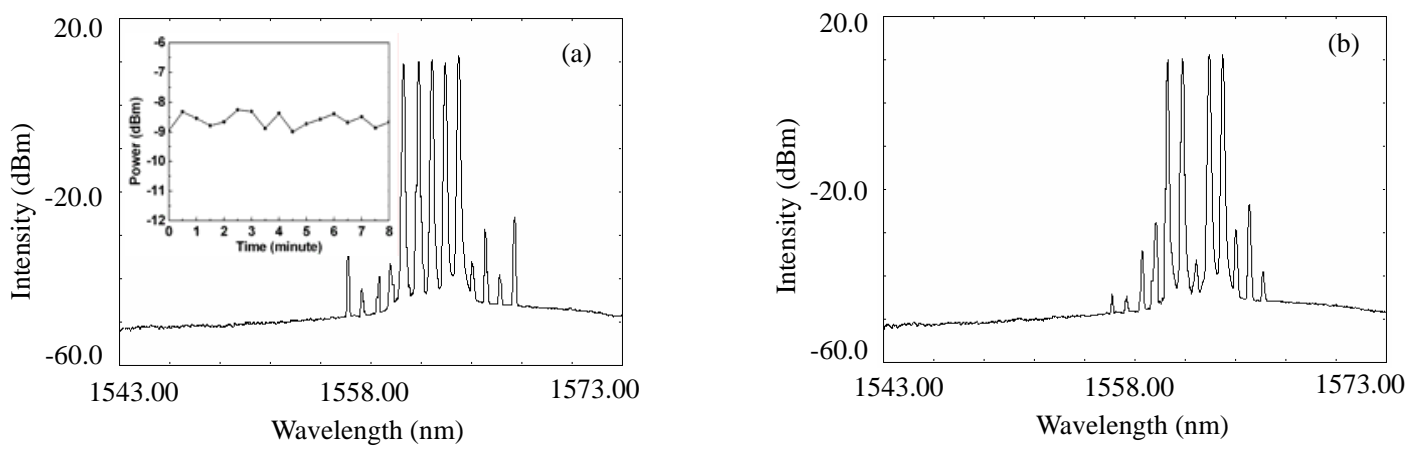

Fig.3 Output spectra of the laser under (a) five-wavelength operation; and (b) four-wavelength operation.

\section{Conclusion}

In conclusion, a simple switchable multiwavelength EDFL using a MM-FBG and HNL-PCF is proposed and demonstrated. The MM-FBG served as a multiwavelength selection element. Stable multiwavelength operation at room temperature can be achieved using the FWM effect induced by the HNL-PCF. The proposed laser can operate in five-wavelength or wavelength switching modes by adjusting the polarization controller in the laser cavity.

\section{Acknowledgment}

This work is supported by the project under Grant G-YX50 of The Hong Kong Polytechnic University. We would like to thank Professor M.S. Demokan for providing the high power EDFA and the PCF.

\section{References:}

[1] Qinghe Mao and John W.Y. Lit, “ Switchable multiwavelength erbium -doped fiber laser with cascaded fiber grating cavities,” IEEE Photon.Technol.Lett. 14, 612-614 (2002)

[2] Xueming Liu, Xiaoqun Zhou, Xiufeng Tang, et al, "Switchable and tunable multiwavelength erbium-doped fiber laser with fiber Bragg gratings and photonic crystal fiber,” IEEE Photon. Technol. Lett. 17 1626-1628 (2005)

[3] Xinhuan Feng, Yange Liu, Shuzhong Yuan, et al, "L-band switchable dual-wavelength erbium-doped fiber laser based on a multimode fiber Bragg grating,” Optics Express 12, 3834-3839 (2004)

[4] Th.Pfeiffer, H.Schmuck, and H.Bulow, "Output power characteristics of erbium-doped fiber ring lasers,” IEEE Photon. Technol. Lett. 4 847-849 (1992)

[5] Kyo Inoue, “ Polarization effect on four-wave mixing efficiency in a single-mode fiber,” IEEE J. Quantum Electron. 28 883-894 (1992) 